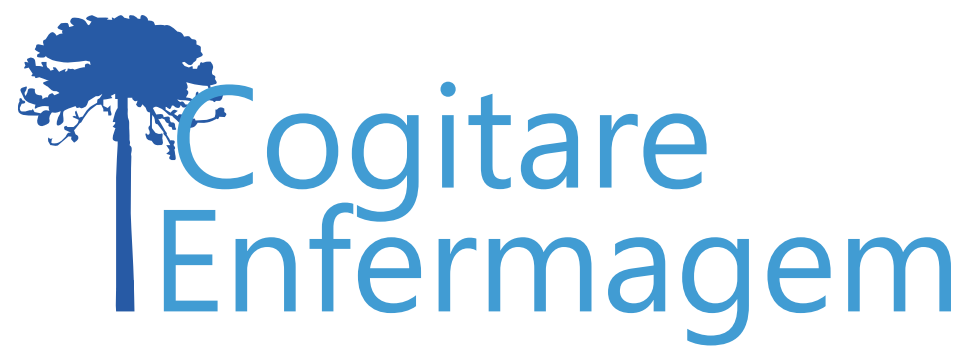

\title{
APLICATIVO PARA AVALIAÇÃO DO NÍVEL DE CONSCIÊNCIA EM ADULTOS: PRODUÇÃO TECNOLÓGICA EM ENFERMAGEM*
}

Wanessa Cristina Tomaz dos Santos Barros ${ }^{1}$, Grace Terezinha Marcon Dal Sasso², Ana Graziela Alvarez ${ }^{3}$, Saulo Fábio Ramos ${ }^{4}$, Sabrina Regina Martins ${ }^{5}$

\section{RESUMO}

Objetivo: desenvolver um aplicativo para a avaliação do nível de consciência em adultos.

Método: produção tecnológica, estruturada a partir do referencial teórico da metodologia da aprendizagem baseada em problemas e desenvolvido considerando os passos do design instrucional contextualizado (análise, design, desenvolvimento, avaliação). Estudo realizado na Universidade Federal de Santa Catarina, de maio de 2014 a abril de 2015.

Resultados: o aplicativo apresenta os conteúdos: escalas para avaliação do nível de consciência, avaliação pupilar, reflexos e padrão respiratório, apresentados aos usuários por meio de textos curtos com breves explicações, imagens e vídeos.

Conclusão: o aplicativo pode facilitar o estudo sobre a temática em qualquer hora ou local, permitindo inclusive sua aplicação à beira do leito, contribuindo assim para melhorias do ensino, assistência e segurança de paciêntes em estado crítico. O resultado da avaliação da tecnologia por docentes e estudantes será objeto de estudos futuros.

DESCRITORES:Informática emenfermagem; Tecnologiabiomédica; Desenvolvimento Tecnológico; Transtornos da consciência; Enfermagem.

*Artigo extraído da tese de doutorado "Aplicativo Móvel para Aprendizagem da Avaliação do Nível de Consciência em Adultos (OMAC)". Universidade Federal de Santa Catarina, 2015.

COMO REFERENCIAR ESTE ARTIGO:

Barros WCTS, Dal Sasso GTM, Alvarez AG, Ramos SF, Martins SR. Aplicativo para avaliação do nível de consciência em adultos: produção tecnológica em enfermagem. Cogitare enferm. [Internet]. 2019 [acesso em "colocar data de acesso, dia, mês abreviado e ano"]; 24. Disponível em: http://dx.doi.org/10.5380/ce.v24i0.60338.

Este obra está licenciado com uma Licença Creative Commons Atribuição 4.0 Internacional.

${ }^{1}$ Enfermeira. Doutora em Enfermagem. Docente da Universidade Federal do Rio Grande do Norte. Santa Cruz, RN, Brasil. (1)

${ }^{2}$ Enfermeira. Doutora em Enfermagem. Docente da Universidade Federal de Santa Catarina. Florianópolis, SC, Brasil. $(2)$ ${ }^{3}$ Enfermeira. Doutora em Enfermagem. Docente da Universidade Federal de Santa Catarina. Florianópolis, SC, Brasil. $(2)$

${ }^{4}$ Enfermeiro. Mestre em Enfermagem. Enfermeiro Assistencial no Hospital Governador Celso Ramos. Florianópolis, SC, Brasil. (2)

${ }^{5}$ Enfermeira. Mestranda em Enfermagem. Universidade Federal de Santa Catarina. Florianópolis, SC, Brasil. $\odot$ 


\title{
APP TO EVALUATE THE LEVEL OF CONSCIOUSNESS IN ADULTS: TECHNOLOGICAL PRODUCTION IN NURSING
}

\begin{abstract}
Objective: to develop an app for assessing the level of consciousness in adults.

Method: technological production, structured from the theoretical framework of problembased learning methodology and developed considering the steps of contextualized instructional design (analysis, design, development, evaluation). Study conducted at the Federal University of Santa Catarina, from May 2014 to April 2015.

Results: the app presents the contents: scales for evaluation of the level of consciousness, pupillary evaluation, reflexes and breathing pattern, presented to the users through short texts with brief explanations, images and videos.

Conclusion: the app can facilitate the study of the theme at any time or place, even allowing its application at the bedside, thus contributing to improvements in teaching, care and safety of critically ill patients. The results of the evaluation of the technology by teachers and students will be the object of future studies.
\end{abstract}

DESCRIPTORS: Information technology in nursing; Biomedical technology; Technological development; Consciousness disorders; Nursing.

\section{APLICACIÓN PARA EVALUACIÓN DEL NÍVEL DE CONSCIENCIA EN ADULTOS: PRODUCCIÓN TECNOLÓGICA EN ENFERMERÍA}

\begin{abstract}
RESUMEN
Objetivo: desarrollar una aplicación para analizar el nivel de consciencia en adultos.

Método: producción tecnológica, estructurada por medio del referencial teórico de la metodología de aprendizaje basada en problemas, la cual se desarrolló considerándo los pasos del diseño educacional contextualizado (análisis, design, desarrollo, evaluación). Estudio que se realizó en la Universidad Federal de Santa Catarina, de mayo de 2014 a abril de 2015. Resultados: la aplicación presenta los contenidos: escalas para evaluación del nivel de consciencia, evaluación pupilar, reflejos y patrón respiratorio, presentados a los usuarios por medio de textos cortos con breves explicaciones, imágenes y videos.

Conclusión: la aplicación puede facilitar el estudio acerca de la temática en cualquier hora o local, posibilitando incluso su uso en el lecho, lo que contribuye para mejorías de la enseñanza, asistencia y seguridad de pacientes en condición crítica. El resultado de la evaluación de la tecnología por docentes y estudiantes será objeto de estudios futuros.
\end{abstract}

DESCRIPTORES: Informática en enfermería; Tecnología biomédica; Desarrollo tecnológico; Trastornos de la consciencia; Enfermería. 
Com a crescente evolução tecnológica, inovações e aprimoramento no âmbito do ensino em enfermagem e na assistência à saúde têm sido exigidos. Assim, observa-se a importância do desenvolvimento de Tecnologias de Informação e Comunicação (TIC), com o intuito de colaborar na aprendizagem e na atualização de conhecimentos. A utilização destas tecnologias trouxe mudanças nas formas de ensinar, aprender e cuidar(1).

Dentre os fatores impulsionadores dessas mudanças, tem-se o rápido avanço tecnológico, principalmente, dos objetos móveis. Em uma pesquisa realizada pelo Instituto Brasileiro de Geografia e Estatística, constatou-se que 92,1\% da população brasileira utilizam smartphones como principal fonte de acesso às informações ${ }^{(2)}$.

Atrelado a esta realidade, destaca-se também o uso de aplicativos móveis que abrangem múltiplas utilidades, sendo estudado, desenvolvido e utilizado pelos profissionais da saúde. Essas ferramentas possibilitam a melhoria da capacidade dos usuários para implementar evidências, estimular o raciocínio clínico e o pensamento crítico(3). $^{(3)}$.

As razões explícitas do aumento da utilização de aplicativos pelos profissionais de saúde relacionam-se à segurança do paciente, pela disponibilização de conhecimentos de forma rápida e abrangente, com diretrizes clínicas atuais, o que pode auxiliar na deliberação de suas intervenções e aprimorar a assistência em saúde ${ }^{(4)}$.

Considera-se que o impacto da tecnologia portátil mudou a maneira de o indivíduo interagir, também em seu contexto social, tendo reflexos no ensino-aprendizagem teórico e prático. Sendo assim, a incorporação de aplicativos nas vivências de docentes e discentes possui efeitos inovadores com métodos educacionais que complementam e associam teoria e prática(5-6).

Os profissionais enfermeiros e os estudantes de enfermagem no âmbito hospitalar encontram dificuldades em relação à busca de conhecimentos pela extensa carga de trabalho, bem como a impossibilidade de participação de práticas em educação permanente. Considerando este contexto, o uso de aplicativos móveis é importante ferramenta para estimular a realização de práticas baseadas em evidências, promover o pensamento crítico, a disseminação de conhecimentos, o auxílio na resolução de problemas, a integração teoria e prática, bem como a interação entre equipe e professores ${ }^{(7)}$.

Os benefícios do uso de aplicativos móveis, seu crescimento nacional e internacional, é evidenciado pelas pesquisas recentes. Ressalta-se o interesse dos profissionais de enfermagem em recursos tecnológicos, para aperfeiçoar a assistência à saúde e promover a qualidade desta assistência ${ }^{(8)}$.

A avaliação do nível de consciência faz parte do atendimento ao paciente grave, logo, torna-se imprescindível que os profissionais tenham conhecimento sobre esta temática. Assim, é de suma importância para que a assistência em enfermagem seja segura e efetiva, a construção de tecnologia móvel que permita a análise das evoluções do paciente assistido, o aprimoramento do raciocínio clínico e o acesso rápido às informações ${ }^{(9-10)}$.

Assim, buscando compartilhar conhecimentos e fomentar em docentes, estudantes e profissionais de saúde o interesse em produzir aplicativos fundamentados cientificamente e que ajudem a apoiar o cuidado seguro e de qualidade, esse estudo teve como objetivo descrever o desenvolvimento de um aplicativo intitulado OMAC® para a avaliação do nível de consciência em adultos.

\section{MÉTODO}

Estudo de produção tecnológica de acordo com as etapas do Design Instrucional Contextualizado, desenvolvido de maio de 2014 a abril de 2015, junto ao Laboratório de 
Produção Tecnológica em Saúde e Grupo de Pesquisa Clínica, Tecnologias e Informática em Saúde e Enfermagem da Universidade Federal de Santa Catarina (LAPETEC/GIATE/ UFSC).

Para o desenvolvimento da tecnologia, foi estruturada uma equipe composta por uma designer gráfica, dois programadores, uma conteudista especialista na área de neurologia e neurocirurgia e uma revisora.

O aplicativo, Objeto Móvel de Avaliação do Nível de Consciência (OMAC®), foi desenvolvido a partir das etapas do Design Instrucional Contextualizado - DIC (Análise, Design, Desenvolvimento e Avaliação). O DIC se baseia principalmente na aprendizagem eletrônica imersiva, sendo sua ênfase na configuração de ambientes personalizados, de acordo com as unidades de estudo que se pretende abordar, em sintonia com as necessidades individuais de aprendizagem do público alvo ${ }^{(11)}$.

As novas tecnologias podem se tornar instrumento de aprendizagem desde que usadas em associação com novas metodologias de ensino, capazes de criar novas dinâmicas de interação, colaboração e construção do conhecimento. Nesta direção, a m-learning vem sendo considerada uma das mais eficazes ferramentas para melhoria da aprendizagem ativa $^{(12)}$.

Para alcançar o objetivo proposto, o referencial teórico da metodologia da Aprendizagem Baseada em Problemas (ABP) foi adotado como suporte ao desenvolvimento do aplicativo (Quadro 1).

Quadro 1 - Etapas da ABP e o desenvolvimento do OMAC®. Florianópolis, SC, Brasil, 2018

\begin{tabular}{|l|l|}
\hline Passos da ABP & Desenvolvimento do aplicativo \\
\hline 1- Identificação do problema & $\begin{array}{l}\text { Revisão bibliográfica - Importância da avaliação do nível de } \\
\text { consciência }\end{array}$ \\
\hline 2- Exploração dos conhecimentos prévios & $\begin{array}{l}\text { Revisão bibliográfica - Identificação das necessidades de } \\
\text { aprendizagem }\end{array}$ \\
\hline $\begin{array}{l}\text { 3- Criação de Hipóteses e possíveis } \\
\text { mecanismos de atuação }\end{array}$ & $\begin{array}{l}\text { Escolha do aspecto da avaliação do nível de consciência que } \\
\text { será visto. Seguir uma sequência ou escolha aleatória }\end{array}$ \\
\hline $\begin{array}{l}\text { 4- Identificação de conteúdos de } \\
\text { aprendizagem }\end{array}$ & $\begin{array}{l}\text { Possibilidade de envio para o professor dos "passos" dos } \\
\text { estudantes durante o uso do aplicativo }\end{array}$ \\
\cline { 1 - 2 } 5- Estudo individualizado & Disponibilidade de acesso em qualquer hora e lugar \\
\hline $\begin{array}{l}\text { 6- Reavaliação e aplicação do novo } \\
\text { conhecimento no problema }\end{array}$ & $\begin{array}{l}\text { Possibilidade de implantar respostas e incentivos durante a } \\
\text { utilização do aplicativo }\end{array}$ \\
\cline { 1 - 2 } 7- Avaliação e reflexão da realidade & \\
\hline
\end{tabular}

Fonte: Adaptado de Walsh ${ }^{(13)}$

Como uma metodologia ativa de ensino-aprendizagem, em que a aprendizagem ocorre pela ação, pelo aprender fazendo, a ABP prevê que o cenário de aprendizagem deve ser escolhido a partir de um contexto real, que faça parte da vida dos estudantes, para que assim ocorra identificação imediata do problema, motivando-os a continuar no desenvolvimento da atividade investigativa. Assim, a ABP permite que o aprendiz adquira graus crescentes de autonomia, por meio da disponibilização de um processo educativo centrado em quem aprende, de modo autodirigido, favorecendo a articulação entre teoria e prática(14). 
O estudo faz parte do macroprojeto intitulado mApp® - Plataforma móvel aberta para desenvolvimento de sistemas $\mathrm{m}$-Saúde na inovação do cuidado humano, aprovado pelo Comitê de Ética em Pesquisa em Seres Humanos, com o número de parecer 711.533.

\section{RESULTADOS}

\section{Etapa de Análise}

$\mathrm{Na}$ primeira etapa, foram identificadas as necessidades de aprendizagem dos estudantes de graduação em enfermagem e as possíveis contribuições para sua solução do problema. Assim, considera-se que um aplicativo, com boa usabilidade e fundamentado em literatura científica, pode facilitar o aprendizado sobre a avaliação de consciência. Para tanto, efetuou-se a busca bibliográfica aprofundada e a definição do conteúdo a ser inserido no aplicativo.

Para definir a estrutura do conteúdo, buscou-se fundamentação na aprendizagem significativa, contextualizada e orientada para o uso das TICs e o desenvolvimento de habilidades para resolver problemas e conduzir projetos nos diversos segmentos da aprendizagem do estudante, aplicando a ABP.

\section{Etapa de Design}

Durante esta etapa, foi definida a estrutura da interface do aplicativo, a sequência do conteúdo, definição de tópicos e seleção de mídias. Assim, o objetivo da etapa foi transformar o conteúdo em material didático, compatível com o formato de aplicativo, de modo atrativo e inovador, utilizando figuras, vídeos e outros recursos.

A elaboração do conteúdo ocorreu após revisão bibliográfica sobre a avaliação do nível de consciência em adultos, que foi estruturado no aplicativo a partir das etapas da ABP. Os conteúdos representam os tópicos que compõem a estrutura do aplicativo, que podem ser acessados de maneira independente, conforme a escolha do usuário, sem prérequisitos (Figura 1 ).

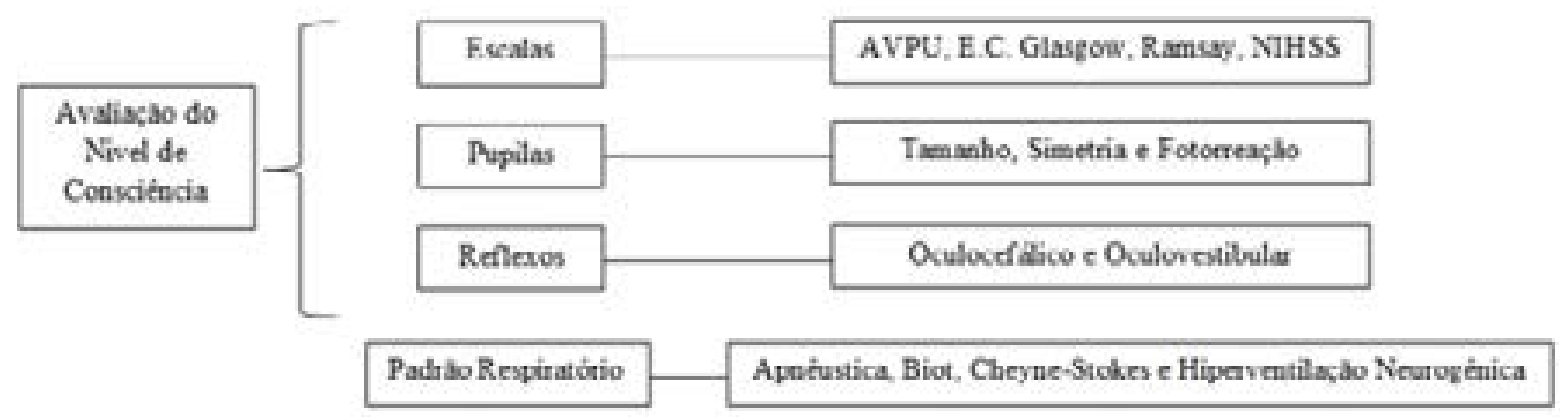

Figura 1 - Estrutura dos dados do OMAC®. Florianópolis, SC, Brasil, 2018

A partir da tela inicial do aplicativo, é possível abrir cada item de avaliação, com as respectivas informações subsequentes. Em cada tela foram disponibilizados ícones demarcando opções a serem marcadas, como no uso das escalas, em que é possível aplicálas e gerar um escore para análise do significado clínico.

No aplicativo, utilizou-se como parâmetro para a avaliação do nível de consciência as escalas de nível de consciência, avaliação pupilar, de reflexos e do padrão respiratório, de acordo com referenciais teóricos da área, conforme apresentado na Figura $2^{(15-20)}$. 

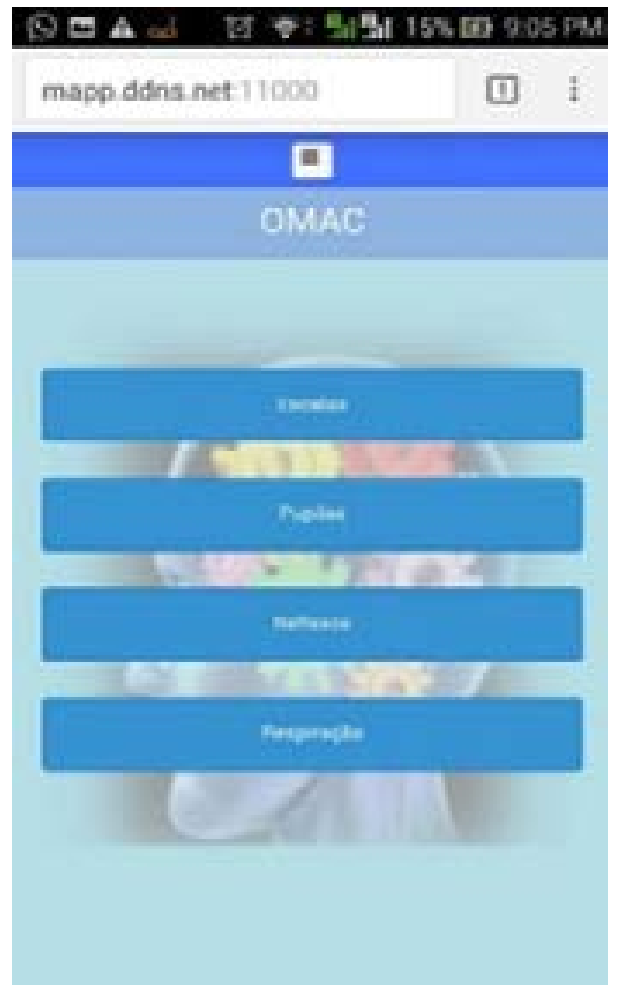

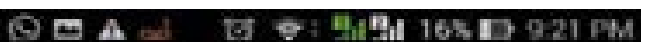

mapp ddns net 11000

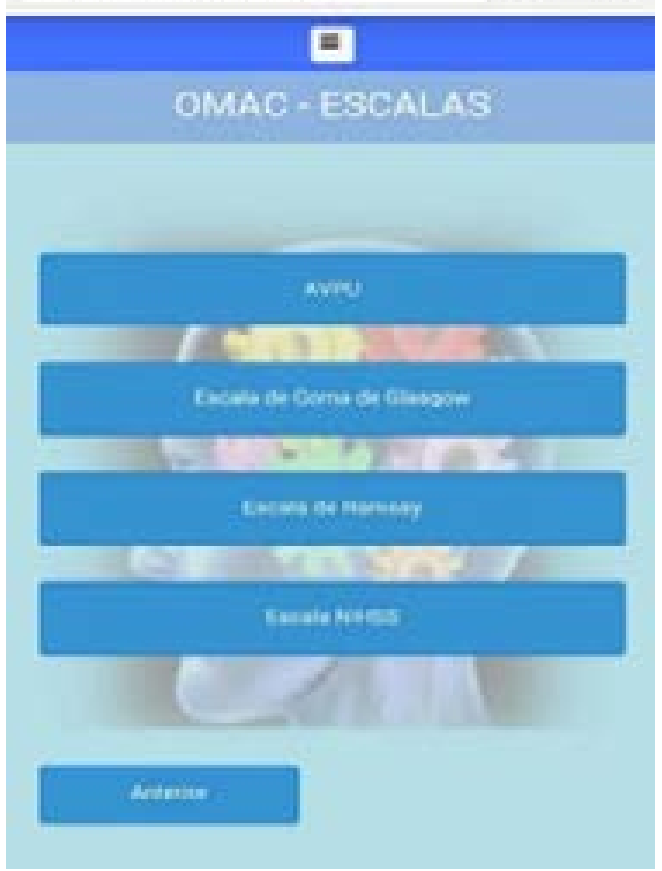

Figura 2 - Etapas e escalas de avaliação do nível de consciência no OMAC®. Florianópolis, SC, Brasil, 2018

A discussão e escolha do projeto gráfico ocorreram nesta fase. Optou-se pela utilização de textos curtos com breves explicações, imagens e vídeos. Os vídeos utilizados são curtos e demonstram situações que exigem a compreensão de alguns itens, como a decorticação, descerebração, reação fotomotora da pupila, reflexos oculocefálico e oculovestibular. Para demonstração dos padrões respiratórios, foram utilizados gráficos animados, que podem auxiliar na localização da lesão que levou a alteração do nível de consciência (Figura 3).

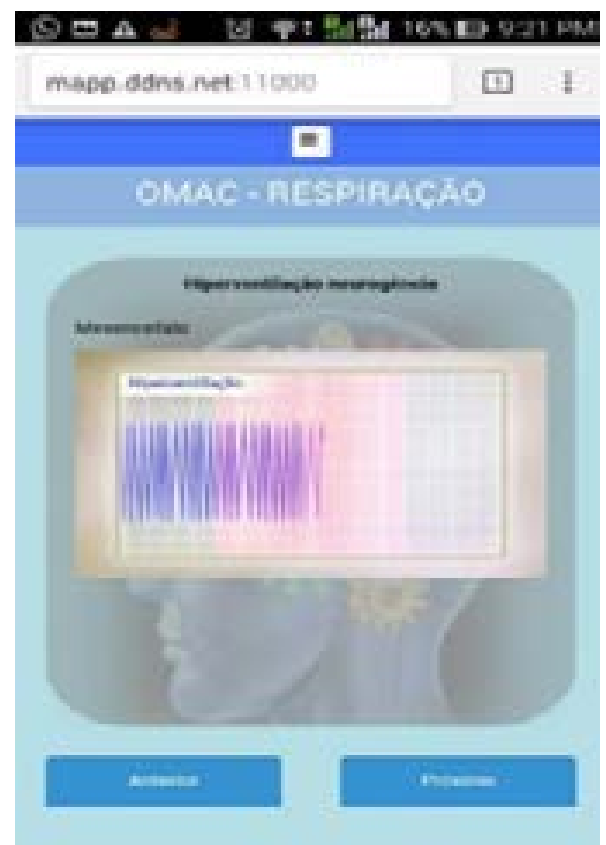

Figura 3 - Padrão de hiperventilação apresentado no OMAC®. Florianópolis, SC, Brasil, 2018 


\section{Etapa de Desenvolvimento}

Nesta etapa ocorreu a produção do aplicativo na plataforma m-App® propriamente dita, considerando-se os pressupostos da metodologia da ABP.

Primeiramente, um storyboard foi criado para conduzir a formulação da sequência do conteúdo do aplicativo e seus respectivos nós críticos antes de sua inserção na plataforma de desenvolvimento $m$-App®, desenvolvida pelo LAPETEC/GIATE/UFSC. Entende-se como nós críticos de aprendizagem para a avaliação do nível de consciência as escalas, pupilas, reflexos e respiração.

A seguir, o conteúdo foi distribuído em diferentes telas, sendo disponibilizadas aos usuários as opções de voltar para a tela anterior, seguir ou voltar para tela principal.

A aplicação foi escrita em uma linguagem leve, interpretada, fundamentada em objetos, e dinẩmica, daí a escolha do JavaScript (JS). Também foi utilizada a biblioteca ExJS, bastante utilizada para aplicações web, cuja escolha se deu pela possibilidade de melhor aproveitamento dos recursos oferecidos pelo padrão HTML5, que atende as mais recentes multimídias ${ }^{(21)}$.

Para definir estilos e efeitos aplicados, foi utilizado o CSS3, enquanto o aplicativo foi estruturado no formato de objeto. A notação utilizada para este objeto foi a JavaScript Object Notation- Notação de Objetos JavaScript, que se baseia em um subconjunto da linguagem de programação JavaScript e é uma formatação leve de troca de dados, que permite às máquinas fácil interpretação e geração de dados e, aos humanos, a facilidade de leitura e escrita. A aplicação está funcionando em NodeJS, o que permite diversas conexões simultâneas, numa única máquina física, ou seja, vários usuários podem acessar o aplicativo sem que haja indisponibilidade de serviços ${ }^{(22-23)}$.

Para que o aplicativo pudesse funcionar em celulares, foi utilizada a Application Programming Interface Cordova, uma interface de programação de aplicativos que permite que o desenvolvedor de aplicativo móvel acesse a função de dispositivo nativo, tais como câmera, GPS e SMS ${ }^{(24)}$.

\section{Etapa de Avaliação}

Na última fase, ocorreu a revisão do conteúdo do aplicativo pelos pesquisadores, a realização de testes para identificação e correção de erros na produção tecnológica, seguido da conferência do projeto gráfico, visando diminuir a possibilidade de falhas.

O aplicativo foi submetido à avaliação por docentes especialistas no cuidado ao paciente grave e também aos alunos do curso de graduação em Enfermagem, realizando um pré e pós-teste, obtendo resultados significativos que serão divulgados em estudos posteriores.

\section{DISCUSSÃO}

A criação de tecnologias inovadoras de cuidado em saúde é destaque no cenário mundial, promovendo qualidade da assistência, visando o raciocínio clínico, condutas baseadas em evidências, informações rápidas e efetivas e a segurança do paciente.

$\mathrm{Na}$ atualidade, observa-se grande crescimento do uso de aplicativos por estudantes e profissionais, sendo que os usos destas tecnologias móveis favorecem a assistência de enfermagem à beira do leito, a comunicação e a interação com o paciente, as habilidades clínicas e de educação em saúde. Apesar dos avanços, é imprescindível a contínua avaliação destes recursos tecnológicos para que as funcionalidades sejam relevantes e que tragam conhecimento atualizado(25). 
A prática da avaliação do nível de consciência é fundamental para monitorização, execução de intervenções e decisão de diagnósticos de enfermagem e médico, sendo prática diária na assistểncia de pacientes graves. No entanto, muitas vezes, essa avaliação é feita por julgamentos que tem conceitos definidos de forma imprecisa e por isso estão condicionados a uma avaliação subjetiva. A demora em detectar sinais de alteração do nível de consciência pode induzir a erros de diagnóstico e ter consequências adversas, como o término prematuro do tratamento e a perda da oportunidade clínica para a continuação do tratamento(20).

Nesse contexto, as escalas e outros requisitos do exame neurológico, utilizados na avaliação do nível de consciência de pacientes graves, representam importante progresso para garantia de assistência e terapêutica adequadas para cada caso. Para isso, é necessária a existência de instrumentos de fácil aplicação, capazes de facilitar a identificação rápida de disfunções ou mudanças nos parâmetros neurológicos ${ }^{(10)}$.

As escalas mais utilizadas e, por isso, escolhidas para inserção no aplicativo foram a AVPU, do inglês, Alert (alerta), Verbal stimuli response (resposta ao estímulo verbal), Painful stimuli response (resposta ao estímulo doloroso) or Unresponsive (irresponsivo), recomendada pelo National Association of Emergency Medical Technicians e Comitê do Trauma do Colégio Americano de Cirurgiões (ACS/COT). A escala pode ser utilizada para fazer avaliação rápida da consciência ${ }^{(18)}$.

Em caso de sedação, a indicação principal é a utilização da escala de Ramsay, que se baseia em seis estágios, sendo os três menores referentes aos, pacientes acordados, enquanto que os valores máximos dizem respeito aos sedados. É considera rápida de aplicar e com fácil interpretação(26).

Outra escala adotada foi a Escala de Acidente Vascular Cerebral do Nacional Institute of Health Stroke Scale, já que as doenças cerebrovasculares podem acarretar importantes alterações do nível de consciência. A escala é simples, segura e baseia-se em 11 itens do exame neurológico ${ }^{(19)}$.

A escala de coma de Glasgow é reconhecida internacionalmente para a avaliação do nível de consciência de pacientes em cuidados intensivos. Os parâmetros dessa ferramenta contribuem para a execução de prática e intervenções clínicas adequadas. Porém, estudos revelam que, apesar da significativa relevância dessa escala, em alguns cenários encontram-se divergência nos resultados dos escores, e consequentemente, contraposições na avaliação clínica. Sendo assim, ter uma tecnologia de fácil acesso, com informações atualizadas, promove avaliação efetiva e concordante ${ }^{(20)}$.

Em nova atualização definiu-se uma nova avaliação clínica na Escala de Coma de Glasgow, sendo analisados pelo item denominado "olhos", a capacidade da abertura ocular pelo som, pressão ou a incapacidade da abertura ocular. No item "verbal" as variáveis possibilitam ao profissional analisar se a fala está orientada, confusa, emana apenas palavras, sons, ou nenhum som emitido. A avaliação motora avalia se o indivíduo obedece aos comandos, à localização, a normal e anormal flexão, extensão ou a incapacidade de movimento ${ }^{(27)}$.

Um dos componentes do exame físico realizado pelo enfermeiro é a avaliação pupilar, em que é possível identificar possíveis danos estruturais no sistema nervoso central, como o aumento da pressão intracraniana, favorecendo assim intervenções imediatas para evitar danos. A avaliação pupilar é composta pela análise do tamanho das pupilas (mióticas, midriáticas, normais), simetria (isocóricas, anisocóricas) e reação fotomotora(27).

Apesar da importância da avaliação comprovada, um estudo internacional verificou que os enfermeiros críticos e neurocirúrgicos subestimam e avaliam incorretamente a reatividade da pupila, sendo assim, ressalta-se a importância de ferramentas de avaliação para aumentar a precisão e consistência da avaliação, para a detecção precoce de problemas neurológicos ${ }^{(28)}$. 
Os reflexos constituem respostas involuntárias a um estímulo, envolvendo um receptor, a via aferente ou sensitiva que leva ao estímulo para o SNC, a presença de neurônios entre as vias aferentes e eferentes e um órgão efetor. Esse mecanismo constitui o funcionamento do reflexo oculocefálico, permite a observação do movimento direcional dos olhos como resposta ao movimento feito pela cabeça. A ocorrência desse estímulo envolve o nervo vestibular, especificamente o VIII do nervo craniano e receptor da musculatura cervical. Os nervos envolvidos nas respostas oculares são nervo abducente VI, nervo oculomotor III e nervo troclear $\mathrm{IV}^{(29)}$.

O reflexo oculovestibular permite a avaliação da ausência ou não dos movimentos oculares, com a participação do nervo vestibular, como via aferente, e os nervos abducente VI, oculomotor III, nas vias eferentes ${ }^{(29)}$.

As avaliações dos reflexos constituem prática fundamental para a detecção de morte encefálica, direcionando as condutas apropriadas para a manutenção dos órgãos e tecidos, se for o caso de potencial doador, ou a finalização do tratamento. Além disto, é fundamental a preparação e a responsabilização dos profissionais acerca dos cuidados nos sistemas corporais ${ }^{(9-10)}$.

O responsável pelo mecanismo respiratório é o SNC, especificamente no córtex cerebral, recebe constante informação sobre a concentração de gases e a composição química do sangue proporcionada por receptores especializados denominados quimiorreceptores. Entretanto, a ocorrência de injúrias cerebrais poderá comprometer a sistemática respiratória, tornando inadequada a perfusão de oxigênio celular e tecidual ${ }^{(30)}$.

Com os padrões respiratórios apresentados pelo paciente, é possível identificar a localização da lesão a nível neurológico, por exemplo: Cheyne-Stokes, associado a lesões no diencéfalo e gânglios basais; hiperventilação neurogênica, comprometimento no mesencéfalo; apnêustica, lesões na ponte e Biot, alterações encontradas no bulbo. Com estas constatações, torna-se fundamental a avaliação do enfermeiro para a detecção de anormalidades respiratórias e posterior intervenção( ${ }^{(30)}$.

Destaca-se como limitação a necessidade de publicação da avaliação da tecnologia por docentes e estudantes, para que seja possível sua ampla utilização na prática educativa e assistencial. Além disto, é preciso realizar a atualização do conteúdo da Escala de Coma de Glasgow.

\section{CONCLUSÃO}

Nesse estudo, que relata a produção do aplicativo $O M A C \AA$, a partir da relevância do tema, na etapa de análise foram identificadas as necessidades e nós críticos da aprendizagem para avaliação do nível de consciência, seguida das etapas de design, desenvolvimento e avaliação do aplicativo produzido.

O aplicativo tem caráter inovador pela possibilidade de ser acessado a partir de diferentes dispositivos móveis e plataformas, proporcionar possibilidade de aprendizado sem pressão de tempo ou lugar, e sem que o usuário necessite se afastar do paciente para obter informação segura, revisar uma prática ou aplicar uma escala. Ainda, buscou-se fomentar nos estudantes o interesse em utilizar o aplicativo, como parte do processo de cuidar em enfermagem do paciente grave, com alteração do nível de consciência.

Destaca-se que os aplicativos produzidos por profissionais, ao convergirem evidências científicas, experiência e conhecimento do contexto, têm o potencial de integrarem-se à prática de tal forma que a ferramenta possa tornar-se procedimento de cuidado. Porém, os usuários desta tecnologia devem atentar-se quanto aos indicativos de contaminação, sendo fundamental a higienização do aparelho antes e após o uso.

Neste momento, é necessário inserir a escala de sedação RASS (Richmond Agitation- 
Sedation Scale) por ser mais detalhada e atualizar a escala de coma de Glasgow a partir das mudanças ocorridas em 2018. Futuramente, após o registro do software, o aplicativo será disponibilizado nas lojas de aplicativos, permitindo amplo acesso às instituições de ensino e assistência.

\section{REFERÊNCIAS}

1. Pissaia LF, Costa AEK, Moreschi C, Rampel C. Tecnologias da informação e comunicação na assistência de enfermagem hospitalar. R Epidemiol Control Infec [Internet]. 2017 [acesso em 16 abr 2018]; 7(4).

Disponível em: http://dx.doi.org/10.17058/reci.v7i4.8959.

2. Instituto Brasileiro de Geografia e Estatística (IBGE). Acesso à Internet e à televisão e posse de telefone móvel celular para uso Pessoal. [Internet]. 2015 [acesso em 20 abr 2018]. Disponível em: https:// biblioteca.ibge.gov.br/visualizacao/livros/liv99054.pdf.

3. Oliveira ARF, Alencar MSM. O uso de aplicativos de saúde para dispositivos móveis como fontes de informação e educação em saúde. Rev. Digit. Bibliotecon. Cien. Inf. [Internet]. 2017 [acesso em 18 abr 2018]; 15(1). Disponível em: https://doi.org/10.20396/rdbci.v15i1.8648137.

4. Flynn GAH, Polivka B, Behr JH. Smartphone Use by Nurses in Acute Care Settings. Comput Inform Nurs [Internet]. 2018; [acesso em mar 2018]; 36(3). Disponível em: http://dx.doi.org/10.1097/ cin.0000000000000400.

5. Willemse JJ. Undergraduate nurses reflections on WhatsApp use in improving primary health care education. Curatonis [Internet]. 2015 [acesso em 16 abr 2018]; 38(2). Disponível em: http://dx.doi. org/10.4102/curationis.v38i2.1512.

6. Barbosa TP. Tecnologias digitais: desafios e perspectivas no ensino superior em saúde. Percurso Acadêmico [Internet]. 2016 [acesso em 01 dez 2016]; 6(12). Disponível em: https://doi.org/10.5752/ P.2236-0603.2016v6n12p449.

7. Innocente AP, Cazella SC. O ensino da enfermagem mediante o uso de dispositivos móveis uma revisão integrativa. Em Rede- Revista de educação à distância [Internet]. 2018 [acesso em 22 abr 2018]; 5(1). Disponível em: https://www.aunirede.org.br/revista/index.php/emrede/article/view/275/303.

8. diCarlantônio LFM, Freitas LCM, Fontes DMA, Silva CFB, Santiago LC. Software productionby nurses for use in patientcare. J res fundamcare [Internet]. 2016 [acesso em 18 abr2018]; 8(2). Disponível em: http://dx.doi.org/10.9789/2175-5361.2016.v8i2.4121-4130.

9. Feijo L. Avaliação do estado de consciência: tradução e validação da Escala FOUR [dissertação]. Porto (Portugual): Universidade do Porto; 2015.

10. Livesay SL. The Bedside Nurse: The Foundation of Multimodal Neuromonitoring. Crit Care Nurs Clin North Am [Internet]. 2016 [acesso em 16 abr 2018]; 28(1). Disponível em: http://dx.doi.org/10.1016/j. cnc.2015.10.002.

11. Filatro A. Design instrucional na prática. São Paulo: Pearson Education do Brasil; 2008.

12. Abachi HR, Muhammad G. The impact of m-learning technology on students and educators. Comput Human Behav [Internet]. 2014 [acesso em 22 jul 2014]; (30). Disponível em: https://doi.org/10.1016/j. chb.2013.06.018.

13. Walsh K, Lawless J, Moss C, Allbon C. The development of an engagement tool for practice development. Practice Development in Health Care [Internet]. 2005 [acesso em 02 jan 2015]; 4(3). Disponível em: https://doi.org/10.1002/pdh.7.

14. Souza SC, Dourado L. Aprendizagem baseada em problemas (ABP): um método de aprendizagem inovador para o ensino educativo. Holos [Internet]. 2015 [acesso em 20 jan. 2015]; (5). Disponível em: http://dx.doi.org/10.15628/holos.2015.2880. 
15. Andrade AF, Carvalho RC, Amorim RLO, Paiva WS, Figueiredo EG, Teixeira MJ. Coma e outros estados de consciência. Rev Med [Internet]. 2007 [acesso em 02 jan 2015]; 86(3). Disponível em: http:// dx.doi.org/10.11606/issn.1679-9836.v86i3p123-131.

16. Mendes CL, Vasconcelos LCS, Tavares JS, Fontan SB, Ferreira DC, Diniz LAC, et al. Escalas de Ramsay e Richmond são equivalentes para a avaliação do nível de sedação em pacientes gravemente enfermos. Rev. bras. ter. intensiva [Internet]. 2008 [acesso em 02 jan 2015]; 20(4). Disponível em: http://dx.doi. org/10.1590/S0103-507X2008000400005.

17. Diccini S, Whitaker IY, Cintra EA. Exame neurológico. In: Barros ALB, organizadora. Anamnese e exame físico. São Paulo: Artmed; 2010.

18. National Association of Emergency Medical Technicians (NAEMT). Comitê do PHTLS. Comitê de Trauma do Colégio Americano de Cirurgiões. Atendimento pré-hospitalar ao traumatizado. 7 ed. Rio de Janeiro: Elsevier; 2012.

19. Ministério da Saúde (BR). Secretaria de Atenção à Saúde. Departamento de Atenção Especializada. Manual de rotinas para atenção ao AVC. Brasília: Ministério da Saúde; 2013.

20. Muñana-Rodríguez JE, Ramírez-Elías A. Escala de coma de Glasgow: origen, análisis y uso apropiado. Enfermería Universitaria [Internet]. 2014 [acesso em 10 abr 2015]; 11(1). Disponível em: http://dx.doi. org/10.1016/S1665-7063(14)72661-2.

21. Mozila Developer Network (MDN). Java Script [Internet]. 2015 [acesso em 20 maio 2015]. Disponível em: https://developer.mozilla.org/pt-BR/docs/Web/JavaScript.

22. Java Script Object Notation (JSON). Introdução ao JSON. [Internet]. 2015 [acesso em 20 maio 2015]. Disponível em: http://json.org/json-pt.html.

23. Node.js. Sobre o Node.js. [Internet]. 2018 [acesso em 20 maio 2015]. Disponível em: https://nodejs. org/en/about/.

24. Lopes CEAF, Painho M. Development of a Mobile Solution for Spatial Data Collection using Open Source Technologies. Proc. Technol. [Internet]. 2014 [acesso em 20 mai 2015]; (16). Disponível em: http:// dx.doi.org/10.1016/j.protcy.2014.10.115.

25. George TP, DeCristofaro C, Murphy PF, Sims A. Student Perceptions and Acceptance of Mobile Technology in an Undergraduate Nursing Program. Health care [Internet]. 2017 [acesso em 20 abr 2018]; 5(3). Disponível em: http://dx.doi.org/10.3390/healthcare5030035.

26. Namigar T, Serap K, Esra AT, ÖzgülO, CanÖA, Aysel A, et al. Correlação entre a escala de sedação de Ramsay, escala de sedação-agitação de Richmond e escala de sedação-agitação de Riker durante sedação com midazolam-remifentanil. Braz. J. Anesthesiol.[Internet]. 2017 [acesso em 25 abr 2018]; 67(4). Disponível em: http://dx.doi.org/10.1016/j.bjan.2017.03.006.

27. Teasdale G, Allen D, Brennan P, E McElhinney, Mackinnon L. Forty years on: updating the Glasgow Coma Scale. Nursing Times [Internet]. 2014 [acesso em 18 jun 2018]; 110(42). Disponível em: https:// www.nursingtimes.net/Journals/2014/10/10/n/p//141015Forty-years-on-updating-the-Glasgow-comascale.pdf

28. Kerr RG, Bacon AM, Baker LL, Gehrke JS, Hahn KD, Lillegraven CL, et al. Underestimation of Pupil Size by Critical Care and Neurosurgical Nurses. Am J Crit Care [Internet]. 2016 [acesso em 20 abr 2018]; 25(3). Disponível em: http://dx.doi.org/10.4037/ajcc2016554.

29. Ivamoto HS. Reflexos. Acta Médica [Internet]. 2014 [acesso em 23 abr 2018]. Disponível em: http:// www.actamedica.org. br/publico/noticia.php?codigo=316\&cod menu=316.

30. Andrade AF, Carvalho RC, Amorim RLO, Paiva WS, Figueiredo EG, Teixeira MJ. Coma e outros estados de consciência. Rev Med [Internet]. 2007 [acesso em 22 abr 2018]; 86(3). Disponível em: https:// doi.org/10.11606/issn.1679-9836.v86i3p123-131. 
Recebido: 04/07/2018

Finalizado: 04/02/2019

Autor Correspondente:

Sabrina Regina Martins

Universidade Federal de Santa Catarina

R. Eng. Agronômico Andrei Cristian Ferreira, s/n - 88040-900 - Florianópolis, SC, Brasil

E-mail: sabrinamartins0704@gmail.com

Contribuição dos autores:

Contribuições substanciais para a concepção ou desenho do estudo; ou a aquisição, análise ou interpretação de dados do estudo - WCTSB, GTMDS, AGA

Elaboração e revisão crítica do conteúdo intelectual do estudo - WCTSB, GTMDS, AGA, SFR, SRM

Aprovação da versão final do estudo a ser publicado - WCTSB, GTMDS, AGA, SFR, SRM

Responsável por todos os aspectos do estudo, assegurando as questões de precisão ou integridade de qualquer parte do estudo - WCTSB, GTMDS, AGA, SFR, SRM 